\title{
$C$ reactive protein and chronic obstructive pulmonary disease: a Mendelian randomisation approach
}

\author{
Morten Dahl, 1,2,3,4 Jørgen Vestbo, 3,4,5,6 Jeppe Zacho, 1,2,3,4 Peter Lange, 3,4,5 \\ Anne Tybjærg-Hansen, 2,3,4,7 Børge G Nordestgaard ${ }^{1,2,3,4}$
}

- An additional figure is published online only. To view this file please visit the journal online (http://thorax.bmj.com)

${ }^{1}$ Department of Clinical Biochemistry, Herlev Hospital, Copenhagen, Denmark ${ }^{2}$ The Copenhagen General Population Study, Herlev Hospital, Copenhagen, Denmark ${ }^{3}$ The Copenhagen City Heart Study, Bispebjerg Hospital, Copenhagen, Denmark ${ }^{4}$ Faculty of Health Sciences, University of Copenhagen, Copenhagen, Denmark ${ }^{5}$ Department of Cardiology and Respiratory Medicine, Hvidovre Hospital, Copenhagen, Denmark ${ }^{6}$ Respiratory Medicine Research Group, University of Manchester, Manchester, UK ${ }^{7}$ Department of Clinical Biochemistry, Rigshospitalet, Copenhagen, Denmark

\section{Correspondence to} Børge G Nordestgaard, Professor, Chief Physician, Department of Clinical Biochemistry, Herlev Hospital, Copenhagen University Hospital, Herlev Ringvej 75, DK-2730 Herlev, Denmark;

brno@heh.regionh.dk

Received 13 November 2009 Accepted 7 October 2010 Published Online First 8 November 2010

\begin{abstract}
Background It is unclear whether elevated plasma $\mathrm{C}$ reactive protein (CRP) is causally related to chronic obstructive pulmonary disease (COPD). The authors tested the hypothesis that genetically elevated plasma CRP causes COPD using a Mendelian randomisation design.

Methods The authors measured high-sensitivity CRP in plasma, genotyped for four single nucleotide polymorphisms in the CRP gene, and screened for spirometry-defined COPD and hospitalisation due to COPD in 7974 individuals from the Copenhagen City Heart Study and in 32652 individuals from the Copenhagen General Population Study.
\end{abstract}

Results Elevated plasma CRP $>3 \mathrm{mg} / \mathrm{l}$ compared with $<1 \mathrm{mg} / \mathrm{l}$ was associated with risk estimates of 1.8 and 2.8 for spirometry-based COPD and of 1.6 and 1.8 for hospitalisation due to COPD in the Copenhagen City Heart Study and the Copenhagen General Population Study, respectively. Genotype combinations of the four CRP polymorphisms were associated with up to a $62 \%$ increase in plasma CRP. However, these genotype combinations did not associate with increased risk of COPD or hospitalisation due to COPD in either cohort or in the two cohorts combined. On instrumental variable analysis, a doubling of plasma CRP versus a doubling of genetically elevated CRP resulted in ORs for COPD of 1.27 (95\% Cl 1.25 to 1.30 ) versus 1.01 (0.81 to 1.26$)$ and for COPD hospitalisation of 1.47 (1.43 to 1.51 ) versus 0.82 (0.59 to 1.13 )

Conclusion Although elevated CRP is related to both a diagnosis of COPD and subsequent hospital admission, genetically elevated plasma CRP is not associated with an increased risk of clinical COPD. This suggests that the association between CRP levels and COPD is not causal.

\section{INTRODUCTION}

Chronic obstructive pulmonary disease (COPD) remains the fourth leading cause of death in the World. ${ }^{12}$ The diagnosis of COPD is based on a lung function test showing obstruction of lung airflow, but the disease also comprises airway inflammation, mucociliary dysfunction, structural changes and extrapulmonary effects. New markers that describe these other components of COPD are being explored with the aim to improve the clinical assessment of COPD. ${ }^{3-5}$

One important inflammatory marker in COPD is elevated plasma $\mathrm{C}$ reactive protein (CRP). Small increases in this acute phase reactant are associated with both disease activity and future risk of hospitalisation and death from COPD. ${ }^{6-16}$ Van Durme et al has found that CRP haplotypes do not associate with increased risk of $\mathrm{COPD},{ }^{16}$ but whether CRP is causally related to COPD or solely a marker of the disease activity is not totally clear. ${ }^{17}$ The answer to this question has clinical importance as drugs that specifically lower CRP are being developed. ${ }^{18}$

One approach to testing for causality between CRP and COPD would be to perform an intervention study with a CRP lowering drug. However, an alternative concept termed 'Mendelian randomisation' can also be used. This design can assess gene-related risk factors for causal association with clinical outcomes. This is because individuals inherit gene variants randomly from their parents. ${ }^{19}$ Thus, genetic variants in the CRP gene that increase plasma concentrations of CRP are an ideal system for testing whether elevated plasma CRP is causally related to COPD.

To test whether genetically elevated plasma CRP is associated with an increased risk of COPD, we have asked the following three questions: (1) is elevated plasma CRP associated with increased risk of COPD, (2) are single nucleotide polymorphisms in the CRP gene associated with increased plasma CRP and (3) is genetically elevated plasma CRP associated with increased risk of COPD. To answer these questions, we measured high-sensitivity CRP in plasma, genotyped for single nucleotide polymorphisms in the CRP gene, screened for COPD using spirometric criteria and obtained information on hospitalisation due to COPD in 7974 individuals from the Copenhagen City Heart Study and 32652 individuals from the Copenhagen General Population Study. ${ }^{70}$

\section{METHODS}

We studied two independent cohorts of white individuals of Danish descent. The two groups were defined so that no individual appears twice in any of the groups, thus permitting independent findings in each group. The studies were approved by Herlev Hospital and by Danish ethics committees, and were conducted according to the Declaration of Helsinki. Written informed consent was obtained from all participants.

\section{Study cohorts}

The Copenhagen City Heart Study ${ }^{7} 21$ is a prospective study of a cohort of individuals 20 years and older randomly selected from the population of Copenhagen. Data were available from this study on COPD $\left(\mathrm{FEV}_{1} / \mathrm{FVC}<0.7\right.$ and $\mathrm{FEV}_{1}<80 \%$ of predicted, excluding individuals with self-reported asthma) and on rate of hospitalisation 
due to COPD (WHO ICD8: 491-492; ICD10: J41-J44) ascertained from 1976 to 2009. From this study, 10230 participants were included in the present analysis.

The Copenhagen General Population Study ${ }^{20} 22$ is a crosssectional study of individuals 20 years and older selected like those in the Copenhagen City Heart Study, from the population of Copenhagen. Data were available from this study on COPD $\left(\mathrm{FEV}_{1} / \mathrm{FVC}<0.7\right.$ and $\mathrm{FEV}_{1}<80 \%$ of predicted, excluding individuals with self-reported asthma) and on prevalence of COPD hospitalisation (WHO ICD8: 491-492; ICD10: J41-J44) ascertained from 1976 to 2009. From this study, 36036 participants were included in the present analysis. We did not have longitudinal data with repeated examinations for the Copenhagen General Population Study and did not perform time-dependent analyses for this cohort.

\section{Genotyping, biochemical analyses and other covariates}

Blood samples were routinely collected for the two source studies. The ABI PRISM ${ }^{\circledR} 7900$ HT Sequence Detection System (Applied Biosystems Inc., Foster City, California, USA) was used to genotype for four single nucleotide polymorphisms in the CRP gene (rs3091244, rs1130864, rs1205, rs3093077). ${ }^{20} \quad 23$ Genotyping was verified by DNA sequencing in $>30$ individuals with each genotype. High-sensitivity CRP was measured using nephelometry or turbidimetry. CRP levels were classified as low $(<1.0 \mathrm{mg} / \mathrm{l})$, average $(1.0-3.0 \mathrm{mg} / \mathrm{l})$, or high $(>3.0 \mathrm{mg} / \mathrm{l})$. If subjects with CRP levels $>10 \mathrm{mg} / \mathrm{l}$ were excluded from the analyses, the results were similar to those presented in this study, except for a significantly decreased OR for COPD hospitalisation among individuals with CRP genotype combination \#9 when combining the two cohorts. Physical activity during leisure time was assessed by The Copenhagen City Heart Study Leisure Time Physical Activity Questionnaire. ${ }^{24}$ Asthma medication was an affirmative answer to the question 'Do you daily take medication for asthma and/or bronchitis?'.

\section{Statistics}

We used Stata for all analyses and NCSS-PASS for power calculations. For trend tests the different groupings of subjects by CRP level, genotype or genotype combination were coded 1, 2, 3, etc, and ranked according to increasing CRP levels. From the four CRP polymorphisms we generated all possible genotype combinations and ranked the nine most common combinations according to increasing plasma CRP levels.

To examine our study hypothesis, we first analysed the relationship between plasma CRP and risk of COPD and hospitalisation due to COPD. Logistic regression estimated ORs for COPD in the Copenhagen City Heart Study and the Copenhagen General Population Study. Cox regression estimated HRs for COPD hospitalisation in the prospective Copenhagen City Heart Study, ${ }^{25}$ and logistic regression estimated ORs for COPD hospitalisation in the Copenhagen General Population Study. The proportional hazard assumption for Cox regression was tested by visual inspection of $\ln (-\ln$ [survival]) versus $\ln$ (analysis time) for all comparisons; no major violations were observed. The models were adjusted for (1) age, sex and smoking or (2) age, sex, smoking, body mass index, physical activity, asthma medication and ischaemic heart disease (ICD8: 410-414; ICD10: I20-I25); the models for hospitalisation due to COPD were also adjusted for $\mathrm{FEV}_{1} \%$ predicted. Overall $\mathrm{p}$ values are test for trend of risk estimates (ORs or HRs) where CRP groups with increasing level of plasma CRP has been coded 1, 2 and 3 .

Second, we analysed the relationship between CRP polymorphisms and polymorphism combinations and plasma CRP in the cross-sectional Copenhagen General Population Study Analyses were performed using Kruskal-Wallis analysis of variance. $p$ Values are for test of trend of mean CRP levels, where CRP genotype/combination groups ranked according to increasing CRP levels have been coded 1, 2, 3 and so forth.

Third, we analysed the relationship between CRP polymorphisms and risk of COPD outcomes. Logistic regression estimated ORs for COPD in the Copenhagen City Heart Study and the Copenhagen General Population Study. Cox regression estimated HRs for COPD hospitalisation in the prospective Copenhagen City Heart Study, ${ }^{25}$ and logistic regression estimated ORs for COPD hospitalisation in the Copenhagen General Population Study. The models were adjusted for (1) age, sex and smoking or (2) age, sex, smoking, body mass index, physical activity, asthma medications and ischaemic heart disease; the models for hospitalisation due to COPD were also adjusted for $\mathrm{FEV}_{1} \%$ predicted. $\mathrm{p}$ Values are for test of trend of mean CRP levels, where CRP genotype/combination groups ranked according to increasing CRP levels have been coded 1, 2, 3 and so forth.

Fourth, the increase in HRs for COPD for a $1 \%$ increase in CRP levels in the Copenhagen City Heart Study was used to predict theoretical HRs for COPD for the change in CRP levels caused by the combined genotypes. Observed and theoretically predicted HRs as a function of plasma CRP levels were corrected for regression dilution bias. ${ }^{26}{ }^{27}$ Logistic regression analysis was used to calculate a combined OR for COPD and hospitalisation due to COPD as a function of genotype for both studies combined.

Finally, the estimated causal OR for COPD in the two cohorts combined was calculated according to Greenland et $a^{2829}$ and compared with the observed $\mathrm{OR}^{30}$; for this purpose, we used generalised least-squares regression.

To address possible misclassification of COPD in younger and older adults from the use of the fixed $\mathrm{FEV}_{1} / \mathrm{FVC}$ threshold, we performed a sensitivity analysis defining COPD based on the fifth percentile lower limit of normal. These analyses using the lower limit of normal definition of COPD yielded similar results to those presented in this study for COPD defined by $\mathrm{FEV}_{1} / \mathrm{FVC}<0.7$ and $\mathrm{FEV}_{1}<80 \%$ of predicted.

\section{RESULTS}

Table 1 presents the distribution of clinical characteristics among the individuals who were free from COPD, had COPD according to spirometry or were hospitalised due to COPD in the Copenhagen City Heart Study and Copenhagen General Population Study. As would be expected, the individuals who had any COPD outcome were more likely males and smokers, were older, and had lower percentage predicted $\mathrm{FEV}_{1}$. More than half of the patients who had spirometry-defined COPD or were hospitalised due to COPD in the Copenhagen City Heart Study did not fulfil the spirometry-based definition of COPD when they entered the study.

\section{Plasma CRP and COPD}

Plasma CRP $>3 \mathrm{mg} / \mathrm{l}$ versus $<1 \mathrm{mg} / \mathrm{l}$ was associated with an $\mathrm{OR}$ of 1.4 (95\% CI 1.1 to 1.9) for COPD and HR of 2.4 (1.6 to 3.6) for hospitalisation due to COPD in the Copenhagen City Heart Study (figure 1). After multifactorial adjustment the equivalent values were $1.8(1.4-2.3)$ and $1.6(1.1-2.5)$, respectively. The $\mathrm{p}$ values for trend were $<0.001$ for all analyses.

Plasma CRP $>3 \mathrm{mg} / \mathrm{l}$ versus $<1 \mathrm{mg} / \mathrm{l}$ was associated with ORs of 1.7 (1.6 to 1.9$)$ and 2.4 (2.1 to 2.9$)$ for COPD and 
Table 1 Characteristics of participants

\begin{tabular}{|c|c|c|c|c|c|c|}
\hline & \multicolumn{3}{|c|}{ Copenhagen City Heart Study } & \multicolumn{3}{|c|}{ Copenhagen General Population Study } \\
\hline & No event & $\begin{array}{l}\text { COPD spirometrically } \\
\text { defined }\end{array}$ & COPD hospitalisation & No event & $\begin{array}{l}\text { COPD spirometrically } \\
\text { defined }\end{array}$ & COPD hospitalisation \\
\hline Women/men & $4221 / 3344$ & $845 / 735$ & $590 / 495$ & $16890 / 14640$ & $1517 / 1575$ & $658 / 577$ \\
\hline Never smokers, \% & 28 & 12 & 5 & 54 & 29 & 19 \\
\hline Ex-smokers, \% & 18 & 12 & 9 & 29 & 32 & 41 \\
\hline Current smokers, \% & 54 & 76 & 86 & 17 & 39 & 40 \\
\hline Plasma CRP & $1.6(1.1-2.7)$ & $1.9(1.3-3.7)$ & $2.4(1.5-5.0)$ & $1.5(1.0-2.6)$ & $2.0(1.2-4.4)$ & $2.5(1.3-5.4)$ \\
\hline Minimum-maximum & $0.1-103$ & $0.1-164$ & $0.1-103$ & $0.1-330$ & $0.1-122$ & $0.1-71$ \\
\hline $\mathrm{FEV}_{1}, \%$ predicted & $94(85-104)$ & $78(68-89)$ & $80(67-93)$ & $92(83-101)$ & $65(55-73)$ & $62(48-79)$ \\
\hline FVC, \% predicted & $93(84-104)$ & $88(77-97)$ & $85(75-97)$ & $93(84-102)$ & $80(70-89)$ & $75(62-88)$ \\
\hline $\mathrm{FEV}_{1} / \mathrm{FVC}$ & $0.83(0.78-0.87)$ & $0.74(0.67-0.81)$ & $0.76(0.70-0.83)$ & $0.79(0.75-0.83)$ & $0.64(0.58-0.67)$ & $0.66(0.56-0.74)$ \\
\hline
\end{tabular}

Values are median (IOR) or percentage. Values for the Copenhagen City Heart Study are pre-outcome values taken prior to the development of COPD. COPD spirometrically defined=FEV $/$ $\mathrm{FVC}<0.7$ and $\mathrm{FEV} 1<80 \%$ of predicted, excluding asthmatics. COPD hospitalisation=ICD8:491-492; ICD10:J41-J44. We used the Copenhagen City Heart Study to test question 1 (CRP level is associated with COPD risk) and question 3 (CRP genotype is associated with COPD risk), and the Copenhagen General Population Study to test question 2 (CRP genotype is associated with CRP level) and retest question 3 .

hospitalisation due to COPD in the Copenhagen General Population Study (figure 1). After multifactorial adjustment the equivalent values were 2.8 (2.4 to 3.1 ) and 1.8 (1.4 to 2.3). The $\mathrm{p}$ values for trend were $<0.001$ for all analyses. The CRP results for COPD hospitalisation in the Copenhagen City Heart Study describe pre-outcome CRP levels, while the Copenhagen General Population Study describes simultaneous or post-outcome levels. The association between CRP levels and risk of COPD, stratified for smoking status, is shown in Supplementary figure 1.

\section{CRP polymorphisms and plasma CRP}

In line with our previous results ${ }^{20}$ the CRP polymorphism rs1205 AA genotype was associated with decreased plasma CRP levels of $24 \%$ compared with the GG genotype (figure 2, Trend $\mathrm{p}<0.001)$. The rs 1130864 TT genotype versus CC genotype was associated with a $25 \%$ increase in plasma CRP level, rs3091244
AA genotype versus CC genotype with a $71 \%$ increase, and rs3093077 GG genotype versus TT genotype with a 55\% increase (all Trend $p<0.001$ ). Combining the genotypes resulted in up to $62 \%$ increase in plasma CRP levels between the highest and lowest levels among the nine most common genotype combinations (figure 2). Partial $\mathrm{r}^{2}$ values for the different CRP polymorphisms ranged from $0.4 \%$ to $1.8 \%$.

\section{CRP polymorphisms and COPD}

The ORs for COPD as a function of genotype in the Copenhagen City Heart Study and the Copenhagen General Population Study did not differ from 1.0 for any of the individual CRP polymorphisms, or for genotype combinations ( $p$ for trend 0.24-0.80; figure 3A). Similarly, the HR for COPD hospitalisation in the Copenhagen City Heart Study and the OR for COPD hospitalisation in the Copenhagen General Population
Figure 1 Risk of chronic obstructive pulmonary disease (COPD) according to plasma levels of $C$ reactive protein (CRP). High-sensitivity CRP level was measured in 8974 individuals participating in the 1991-1994 examination of the Copenhagen City Heart Study and subsequently correlated with spirometry-defined COPD or followed with respect to hospitalisation due to COPD. Highsensitivity CRP level was also measured in 32652 individuals participating in the Copenhagen General Population Study and subsequently correlated with COPD and hospitalisation due to COPD. Multiple adjustment was for age, sex, smoking, body mass index, physical activity, asthma medication and ischaemic heart disease; models for hospitalisation due to COPD were also adjusted for $\mathrm{FEV}_{1} \%$ predicted. Overall $\mathrm{p}$ values are test for trend of risk estimates (ORs or HRs), where CRP groups with increasing level of plasma CRP have been coded 1, 2 and 3 .
Copenhagen City Heart Study $\begin{array}{lcc}\text { COPD } & & \\ \text { CRP, mg/l } & \text { Participants } & \text { Events } \\ <1 & 616 & 79 \\ 1-3 & 4998 & 853 \\ >3 & 1721 & 412\end{array}$ Age, sex, and smoking adjusted p risk p trend Multiple adjusted $\mathrm{p}$ risk $\mathrm{p}$ trend

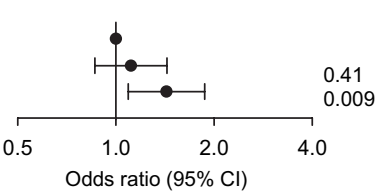

COPD hospitalisation

$\begin{array}{ccc}\text { COPD hospitalisation } & \\ \text { CRP, mg/l } & \text { Participants } & \text { Even } \\ <1 & 593 & 27 \\ 1-3 & 4866 & 471 \\ >3 & 1715 & 302\end{array}$ Odds ratio $(95 \% \mathrm{Cl})$

$<0.001$

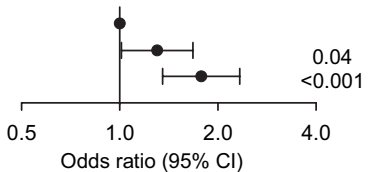

$<0.001$ Odds ratio $(95 \% \mathrm{Cl})$

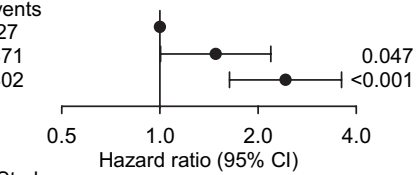

Copenhagen General Population Study COPD

$\begin{array}{ccc}\text { CRP, } \mathrm{mg} / \mathrm{l} & \text { Participants } & \text { Events } \\ <1 & 8912 & 575 \\ 1-3 & 17810 & 1391 \\ >3 & 7888 & 1125\end{array}$

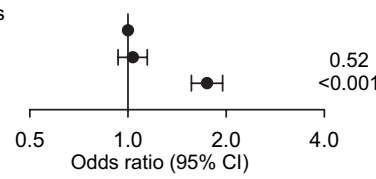

$<0.001$

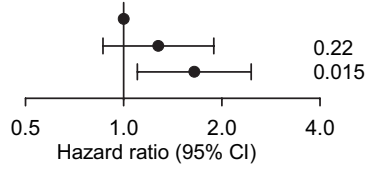

$<0.001$

COPD hospitalisation

CRP, mg/l Participants Events

$\begin{array}{ccc}<1 & 8371 & 188 \\ 1-3 & 16139 & 515 \\ >3 & 6908 & 531\end{array}$

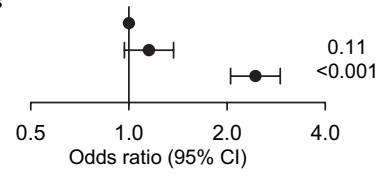

$<0.001$

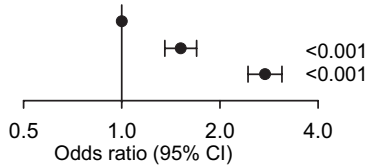

$<0.001$

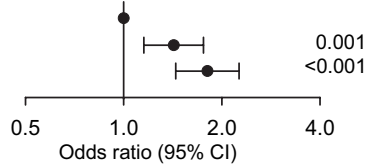


Figure 2 Plasma levels of $C$ reactive protein (CRP) according to CRP genotype and genotype combinations in the Copenhagen General Population Study. High-sensitivity CRP levels and CRP genotyping was determined in 36036 individuals. Partial $r^{2}$ values were after adjustment for variation in plasma CRP levels due to age, gender, smoking and body mass index. p Values are for Cuzick non-parametric test for trend of mean CRP levels, where CRP genotype/combination groups ranked according to increasing CRP levels have been coded 1, 2, 3 and so forth.

\begin{tabular}{|c|c|c|}
\hline \multicolumn{3}{|c|}{$\begin{array}{l}\text { CRP genotypes } \\
\text { rs1205, *1081 G } \rightarrow A\end{array}$} \\
\hline & GG & 15952 \\
\hline & GA & 15945 \\
\hline & AA & 4139 \\
\hline \multicolumn{3}{|c|}{ rs1130864, ${ }^{*} 223 \mathrm{C} \rightarrow \mathrm{T}$} \\
\hline & $\mathrm{CC}$ & 16945 \\
\hline & CT & 15458 \\
\hline & TT & 3633 \\
\hline \multicolumn{3}{|c|}{ rs3091244,-390 C $\rightarrow T \rightarrow A$} \\
\hline & $\mathrm{CC}$ & 14568 \\
\hline & CT & 14348 \\
\hline & TT & 3636 \\
\hline & CA & 2270 \\
\hline & AT & 1140 \\
\hline & AA & 74 \\
\hline \multicolumn{3}{|c|}{$\mathrm{rs} 3093077,{ }^{*} 3678 \mathrm{~T} \rightarrow \mathrm{G}$} \\
\hline & TT & 32594 \\
\hline & GT & 3356 \\
\hline & GG & 86 \\
\hline \multicolumn{3}{|c|}{ CRP genotype combinations } \\
\hline \#1 & AАССССТT & 4078 \\
\hline \#2 & GACCCCTT & 7251 \\
\hline \#3 & GGCCCCTT & 3199 \\
\hline \#4 & GACTCTTT & 7415 \\
\hline \#5 & GGCTCTTT & 6828 \\
\hline \#6 & GACCCAGT & 1175 \\
\hline \#7 & GGTTTTTT & 3595 \\
\hline \#8 & GGCCCAGT & 1050 \\
\hline 79 & GGCTATGT & 1082 \\
\hline
\end{tabular}
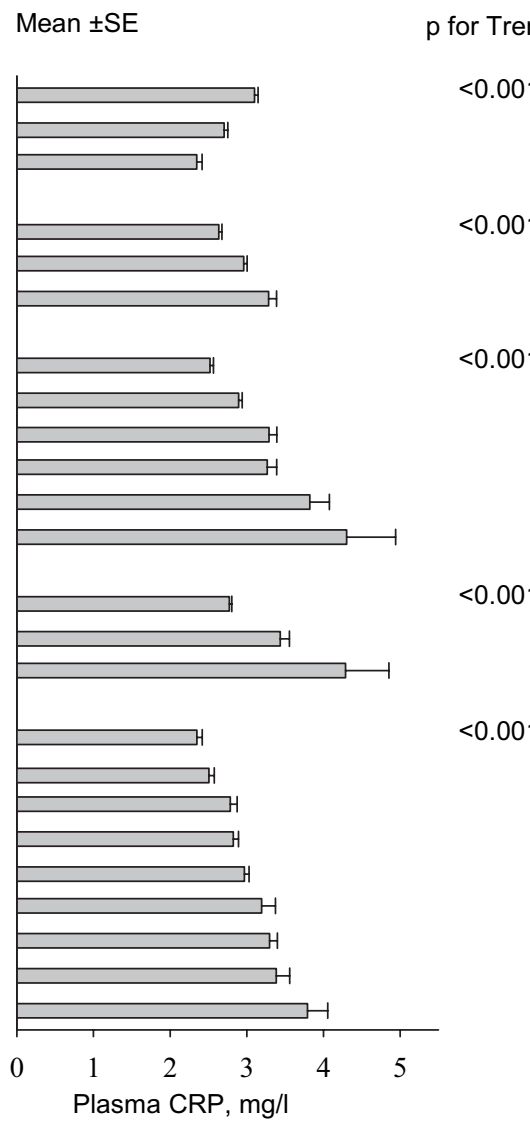

On instrumental variable analysis a doubling of plasma CRP

Study did not differ consistently from 1.0 for any of the individual CRP polymorphisms, or for genotype combinations ( $\mathrm{p}$ for trend $0.12-0.78$; figure $3 \mathrm{~B}$ ).

When analysing the annual decline in $\mathrm{FEV}_{1}$ among individuals with COPD in the Copenhagen City Heart Study, there was no significant difference for any of the individual CRP polymorphisms, or for genotype combinations ( $p$ for trend 0.19 to 0.65 ; figure 4). Partial $\mathrm{r}^{2}$ values for the different CRP polymorphisms ranged from $0.09 \%$ to $0.81 \%$.

\section{Predicted versus observed risk of COPD}

We assumed that genetically elevated plasma CRP levels confer the same OR for COPD as that observed for plasma CRP levels in the general population. Based on this assumption, increments in plasma CRP levels of up to $62 \%$ as a function of CRP genotype combinations theoretically predicted ORs of up to 1.16 (1.09 to 1.23) for COPD and 1.35 (1.28 to 1.42) for hospitalisation due to COPD (figure 5). However, when COPD events and controls from all studies shown in figure 3 were combined to achieve the maximal statistical power, the observed ORs for COPD and hospitalisation due to COPD as a function of genotype combinations did not differ from 1.0 (figure 5). Genotype combinations \#7-9 versus \#1 resulted in an increase in plasma CRP of $45 \%$ and thus theoretically predicted an OR of 1.11 (1.07 to 1.16) for COPD and 1.25 (1.20 to 1.29) for hospitalisation due to COPD (figure 5). However, the observed ORs for COPD and hospitalisation due to COPD for genotype combinations \#7-9 versus \#1 were 1.05 (0.93 to 1.19) and 0.92 (0.77 to 1.10), respectively. Combining all individuals with COPD or hospitalisation due to COPD from the two studies, the \#7-9 versus \#1 genotype combinations had $80 \%$ power to detect ORs of 1.16 for spirometrically defined COPD and 1.22 for hospitalisation due to COPD (figure 5). resulted in an OR of 1.27 (1.25 to 1.30) for COPD, while a doubling of genetically elevated CRP resulted in an OR of 1.01 (0.81 to 1.26) for COPD ( $p=0.04$ for the comparison between the two ORs). Corresponding ORs for COPD hospitalisation were 1.47 (1.43 to 1.51$)$ and 0.82 (0.59 to 1.13$)(p=0.001)$.

\section{DISCUSSION}

To examine whether genetically elevated plasma CRP is causally related to COPD, this study has measured high-sensitivity CRP in plasma, genotyped for variants in the CRP gene, and screened for COPD in 40626 individuals from two ethnically homogenous Danish populations. Three salient findings are provided by the study: (1) plasma CRP above $3 \mathrm{mg} / \mathrm{l}$ is associated with increased risk for clinical COPD, (2) variants in the CRP gene are associated with elevated levels of plasma CRP and (3) analysing this data using a Mendelian randomisation design, we find that genetically elevated plasma CRP is not associated with risk of clinical COPD. This suggests that elevated plasma CRP is not causally related to COPD, but more likely serves as a marker of disease severity in COPD.

Consistent with our results van Durme et al have found that three tagging polymorphisms in the CRP gene (1184, 2042, 2911) are not associated with altered risk of COPD. ${ }^{16}$ In contrast to this, Sunyer et al found that the rs1205 polymorphism in the CRP gene and two haplotypes tagged by the rs1205 and rs1800947 polymorphisms were associated with better lung function. ${ }^{31}$ It is uncertain whether the two previous studies had enough genetic CRP range and study size to examine for causality between CRP and features of COPD. On instrumental variable analysis we now find that a doubling of plasma CRP resulted in an elevated risk for COPD outcomes, while 
A

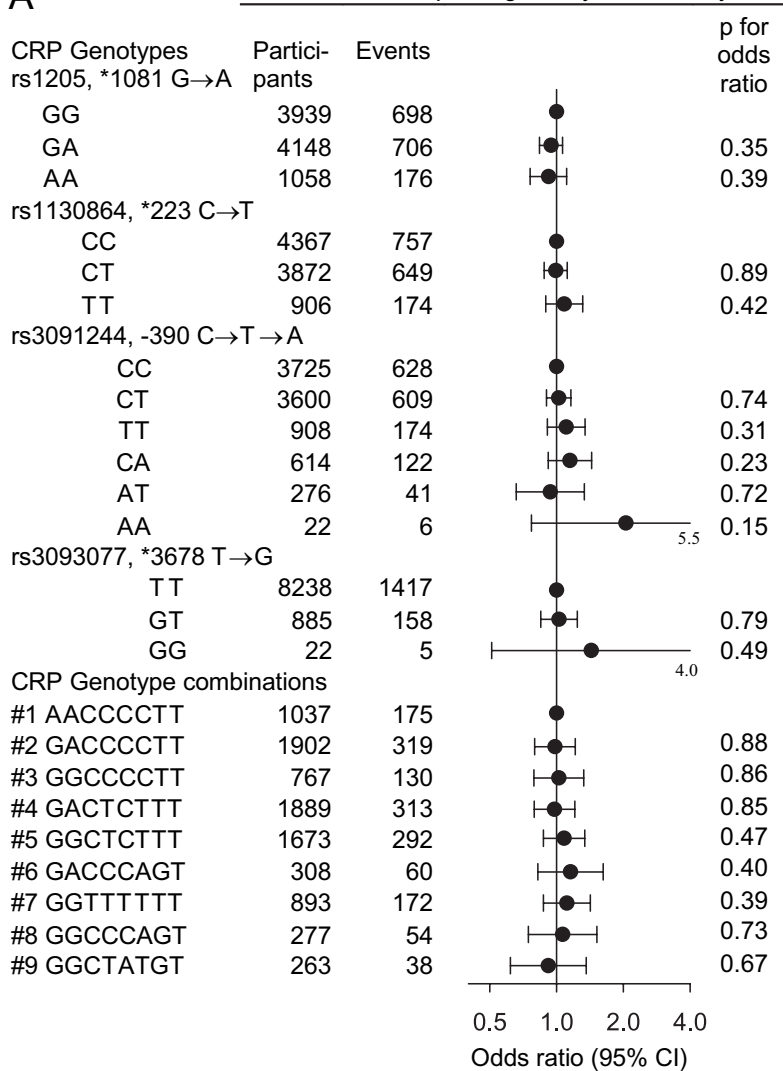

Copenhagen General Population Study

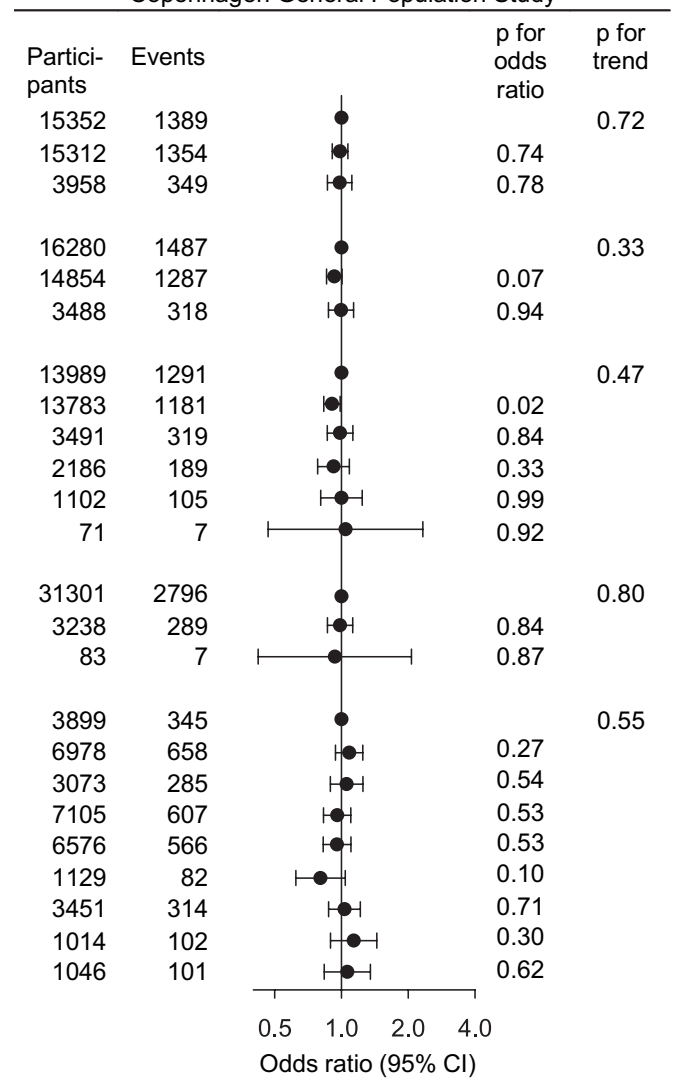

Copenhagen General Population Study

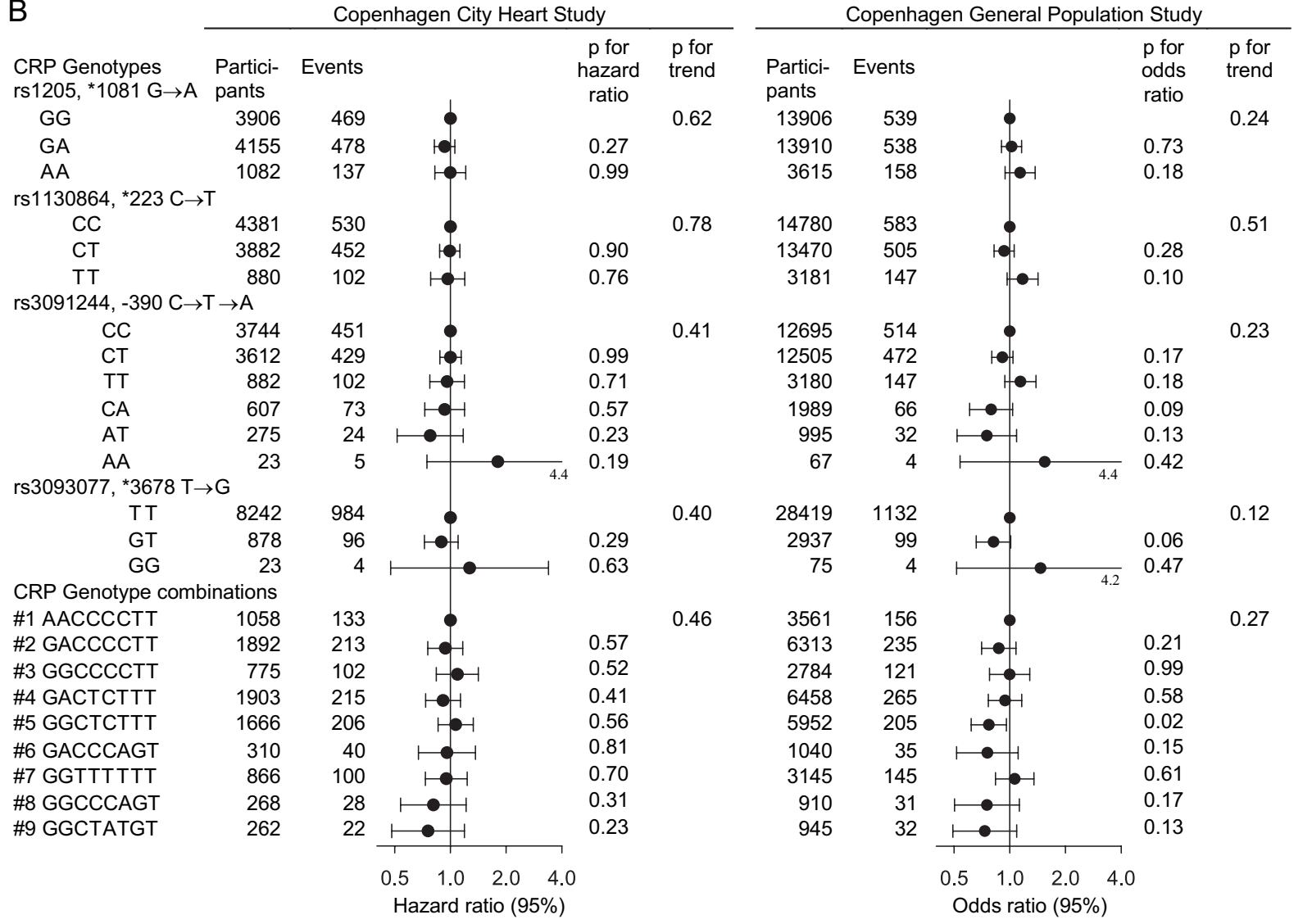

Figure 3 (A, B) Risk of chronic obstructive pulmonary disease (COPD) according to $C$ reactive protein (CRP) genotype and genotype combinations. The ORs and HRs were adjusted for age, sex and smoking. After multiple adjustment for age, sex, smoking, body mass index, physical activity, asthma medication, ischaemic heart disease and/or FEV $\%$ predicted, the risk estimates were similar to those presented. Overall $\mathrm{p}$ values are test for trend of risk estimates (ORs or HRs), where CRP genotype/combination groups ranked according to increasing CRP levels have been coded 1, 2, 3 and so forth. 
Figure 4 Annual $\mathrm{FEV}_{1}$ decline according to $C$ reactive protein (CRP) genotype and genotype combinations among individuals with spirometrydefined chronic obstructive pulmonary disease in the Copenhagen City Heart Study. Annual $\mathrm{FEV}_{1}$ decline and CRP genotyping was determined in 1513 individuals with COPD. Partial $r^{2}$ values were after adjustment for variation in annual $\mathrm{FEV}_{1}$ decline due to age, gender, smoking and body mass index. $p$ Values are for Cuzick non-parametric test for trend of mean $\mathrm{FEV}_{1}$ decline, where CRP genotype/combination groups ranked according to increasing CRP levels have been coded 1, 2, 3 and so forth.

\begin{tabular}{|c|c|c|}
\hline \multicolumn{3}{|c|}{$\begin{array}{l}\text { CRP genotypes } \\
\text { rs1205, *1081 G } \rightarrow A\end{array}$} \\
\hline & GG & 670 \\
\hline & GA & 678 \\
\hline & $\mathrm{AA}$ & 165 \\
\hline \multicolumn{3}{|c|}{ rs1130864, ${ }^{*} 223 \mathrm{C} \rightarrow \mathrm{T}$} \\
\hline & CC & \\
\hline & CT & \\
\hline & TT & \\
\hline \multicolumn{3}{|c|}{ rs3091244,-390 C $\rightarrow \mathrm{T} \rightarrow \mathrm{A}$} \\
\hline & $\mathrm{CC}$ & 594 \\
\hline & CT & 592 \\
\hline & TT & 16 \\
\hline & $\mathrm{CA}$ & 117 \\
\hline & AT & 39 \\
\hline & $\mathrm{AA}$ & \\
\hline \multicolumn{3}{|c|}{ rs3093077, ${ }^{*} 3678 \mathrm{~T} \rightarrow \mathrm{G}$} \\
\hline & TT & 1356 \\
\hline & GT & \\
\hline & GG & \\
\hline \multicolumn{3}{|c|}{ CRP genotype combinations } \\
\hline \#1 & ААССССТТ & 164 \\
\hline \#2 & GACCCCTT & 301 \\
\hline \#3 & GGCCCCTT & 12 \\
\hline \#4 & GACTCTTT & 30 \\
\hline$\# 5$ & GGCTCTTT & 28 \\
\hline \#6 & GACCCAGT & \\
\hline \#7 & GGTTTTTT & 163 \\
\hline \#8 & GGCCCAGT & \\
\hline$\# 9$ & GGCTATGT & \\
\hline
\end{tabular}

政 . 356

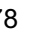
年 . 52
5

$$
0
$$

Mean \pm SE

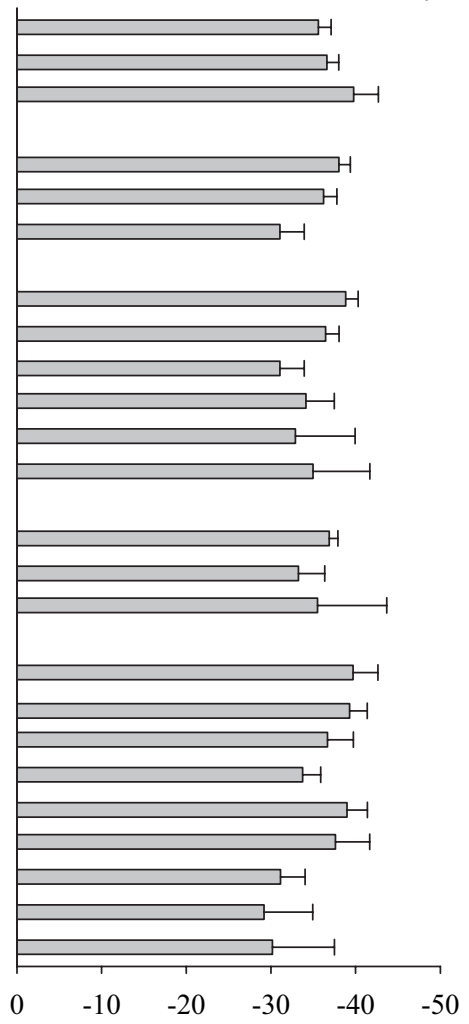

$\mathrm{FEV}_{1}$ decline, $\mathrm{ml} /$ year a doubling of genetically elevated plasma CRP had no relation to COPD. This strongly suggests that the association between CRP levels and COPD is reverse causal or confounded by a third factor like inflammation in general.

Our results do not preclude clinical use for plasma CRP in the assessment of COPD. Plasma CRP may be a marker of prognosis in COPD as small increases are associated with a poor COPD prognosis in the present study and other studies. ${ }^{72}$ Plasma CRP may also be a marker of the efficacy of COPD therapy. Antiinflammatory treatments that are beneficial towards COPD are linked with reduction in plasma CRP level in some $e^{14} 3233$ but not all studies. ${ }^{34}$ It is possible that elevated plasma CRP in combination with other risk markers of lung disease can be used to categorise individuals with low, medium and high risk of future COPD or be used after further validation to guide the efficacy of therapy in COPD. ${ }^{32} 33$

The cause for the increased level of plasma CRP in COPD patients is not totally clear. One hypothesis suggests that plasma CRP is raised as a result of IL-6 production from the inflamed lungs in COPD. Cigarette smoking is the most commonly encountered risk factor for COPD and contributes to both lung inflammation and elevated CRP in COPD patients. Asthma, passive smoking, inhalation of outdoor and indoor air pollutants, obesity and physical inactivity could also cause lung inflammation and raise plasma CRP levels, and this could be particularly relevant among non-smoking COPD patients.

The potential limitations of the study also need to be considered. Mendelian randomisation can be used for the study of causation between modifiable exposures and disease, provided the following six criteria are fulfilled. ${ }^{35}$

1. The presence of suitable genetic variants for the study of the modifiable exposure of interest: the CRP variant rs3091244 modulates transcription factor binding to the CRP gene and is the single most important polymorphism influencing CRP levels, while the CRP variants rs1130864, rs1205 and rs3093077 can be used as tag single nucleotide polymorphisms together describing most of the variation in plasma CRP levels. ${ }^{20}$

2. Reliable genotype-intermediate-phenotype and genotypedisease associations can be established: we showed that the four CRP polymorphisms are associated with elevated plasma CRP levels, but not with risk of COPD in two independent studies analysing data from up to 44359 individuals.

3. There is no confounding of these relationships: linkage disequilibrium could not be detected between the four CRP polymorphisms used in our study and other known nearby genes potentially influencing plasma CRP and/or risk of COPD. ${ }^{20}$

4. There are no pleiotropic effects of the genetic variants of interest: we are not aware of any known association of the four CRP polymorphisms with a phenotype besides changes in CRP level that could influence the risk of COPD.

5. There is no compensation by other genes during development (canalisation) ${ }^{35}{ }^{36}$ : canalisation and compensation by other genes during development are generally difficult to assess; however, we are not aware of any such potential effects.

6. Population admixture that differs between cases and controls: we studied two large and ethnically homogenous populations of Danish descent, and effectively can exclude admixture. Hence, classical limitations of the Mendelian randomisation do not appear to be of major importance in the present study.

A large percentage of CRP variability depends on other nongenetic factors, and this could limit the Mendelian randomisation approach. Genetically determined CRP concentrations 


\begin{tabular}{|c|c|c|c|c|c|c|c|c|c|c|c|}
\hline \multicolumn{2}{|c|}{$\begin{array}{l}\text { CRP genotype } \\
\text { combinations }\end{array}$} & \multirow{2}{*}{$\begin{array}{c}\triangle \mathrm{CRP} \\
0 \%\end{array}$} & \multirow{2}{*}{$\begin{array}{c}\mathrm{N} \\
4078\end{array}$} & \multirow{2}{*}{ Theoretically predicted risk of COPD } & \multicolumn{3}{|c|}{ Observed risk of COPD } & $\begin{array}{l}p \text { for odds } \\
\text { ratio }\end{array}$ & Controls & COPD & \multirow{2}{*}{$\begin{array}{l}80 \% \text { power } \\
\text { Odds ratio }\end{array}$} \\
\hline \#1 & AАСCCCTT & & & & & $\phi$ & & & 4416 & 520 & \\
\hline \#2 & GACCCCTT & $7 \%$ & 7251 & $\bullet$ & & $\mapsto b-1$ & & 0.64 & 7903 & 977 & 1.15 \\
\hline$\# 3$ & GGCCCCTT & $19 \%$ & 3199 & $\bullet$ & & $\mapsto \bullet-1$ & & 0.47 & 3425 & 415 & 1.18 \\
\hline \#4 & GACTCTTT & $20 \%$ & 7415 & ••|ㅓㅏ & & $1-1$ & & 0.40 & 8074 & 920 & 1.15 \\
\hline$\# 5$ & GGCTCTTT & $26 \%$ & 6828 & 너 & & $1-1$ & & 0.61 & 7391 & 858 & 1.15 \\
\hline \#6 & GACCCAGT & $36 \%$ & 1175 & $\vdash \bullet$ & & $\longmapsto-1$ & & 0.59 & 1295 & 142 & 1.25 \\
\hline$\# 7$ & GGTTTTTT & $40 \%$ & 3595 & $\mapsto \bullet-1$ & & $\mapsto b-1$ & & 0.58 & 3858 & 486 & 1.17 \\
\hline$\# 8$ & GGCCCAGT & $44 \%$ & 1050 & $\mapsto-1$ & & $\mapsto \bullet$ & & 0.26 & 1135 & 156 & 1.26 \\
\hline$\# 9$ & GGCTATGT & $62 \%$ & 1082 & $\longmapsto-1$ & & $\longmapsto$ & & 0.91 & 1170 & 139 & 1.26 \\
\hline \multirow{2}{*}{\multicolumn{2}{|c|}{$\begin{array}{l}\# 3-6 \\
\# 7-9\end{array}$}} & $23 \%$ & 18617 & •-1 & & $1-1$ & & 0.63 & 20185 & 2335 & 1.13 \\
\hline & & $45 \%$ & 5727 & $\mapsto-1$ & & $1-1$ & & 0.44 & 6163 & 781 & 1.16 \\
\hline & & & & $\begin{array}{lllll}1.1 & 1.2 & 1.3 & 1.4 & 1.5\end{array}$ & 0.5 & 1.4 & 2.0 & & & & \\
\hline
\end{tabular}

\begin{tabular}{|c|c|c|c|}
\hline \multicolumn{2}{|c|}{$\begin{array}{l}\text { CRP genotype } \\
\text { combinations }\end{array}$} & \multirow{2}{*}{$\begin{array}{c}\triangle \mathrm{CRP} \\
0 \%\end{array}$} & \multirow{2}{*}{$\begin{array}{c}N \\
4078\end{array}$} \\
\hline \#1 & АACCCCTT & & \\
\hline \#2 & GACCCCTT & $7 \%$ & 7251 \\
\hline \#3 & GGCCCCTT & $19 \%$ & 3199 \\
\hline \#4 & GАСTСTTT & $20 \%$ & 7415 \\
\hline \#5 & GGCTCTTT & $26 \%$ & 6828 \\
\hline \#6 & GACCCAGT & $36 \%$ & 1175 \\
\hline \#7 & GGTTTTTT & $40 \%$ & 3595 \\
\hline \#8 & GGCCCAGT & $44 \%$ & 1050 \\
\hline \#9 & GGCTATGT & $62 \%$ & 1082 \\
\hline & & $23 \%$ & 18617 \\
\hline \#7- & & $45 \%$ & 5727 \\
\hline
\end{tabular}

Theoretically predicted risk of COPD
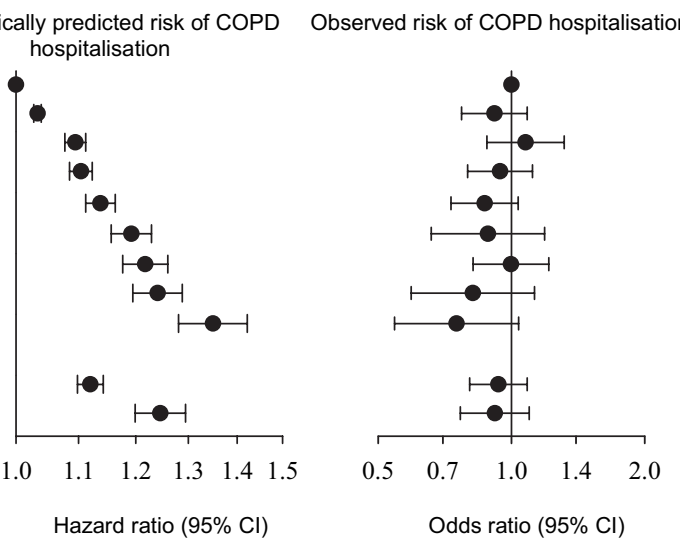

$p$ for odds
ratio

$\begin{array}{ccc}\text { Controls } & \begin{array}{c}\text { COPD } \\ \text { hospitalisation }\end{array} & \begin{array}{c}80 \% \text { power } \\ \text { Odds ratio }\end{array} \\ 4330 & 289 & - \\ 7757 & 449 & 1.21 \\ 3336 & 223 & 1.26 \\ 7881 & 480 & 1.21 \\ 7207 & 411 & 1.21 \\ 1275 & 75 & 1.36 \\ 3766 & 245 & 1.25 \\ 1119 & 59 & 1.38 \\ 1153 & 54 & 1.38 \\ & & \\ 19699 & 1189 & 1.19 \\ 6038 & 358 & 1.22\end{array}$

Hazard ratio $(95 \% \mathrm{Cl})$

Odds ratio $(95 \% \mathrm{Cl})$

Figure 5 Predicted versus observed risk of chronic obstructive pulmonary disease (COPD) as a function of $C$ reactive protein (CRP) genotype combination. The OR used for calculating the theoretically predicted risk of COPD was adjusted for age, sex, and smoking status. The ORs for the observed risk of COPD were adjusted for age, sex and smoking status. In order to achieve maximal statistical power, we combined all individuals with the nine most common CRP genotype combinations from the Copenhagen City Heart Study and the Copenhagen General Population Study (4613 COPD cases vs 38667 controls and 2285 individuals hospitalised due to COPD vs 37824 controls). The right column shows the 0 R at one-sided $p<0.05$ that can be detected in these combined studies at $80 \%$ statistical power.

varied less than plasma CRP levels. It could be that plasma CRP causes COPD or protects from the disease at CRP levels beyond those determined by the CRP genotype data. However, there is a linear relationship between levels of CRP and risk of COPD in this study and there was sufficient statistical power to exclude a causal association between plasma CRP and COPD even within the limited plasma CRP range determined by the genotype data (figures 3 and 5). CRP is an acute phase reactant and was only measured once in our two cohorts. However, the results of the two studies were similar and when analysing only those subjects with $\mathrm{CRP}<10 \mathrm{mg} / 1$ the results were similar to those presented in this study.

We used both cross-sectional and prospective study designs to confirm our data in two independent cohorts and to achieve maximal statistical power for assessing a causal relationship between CRP and COPD. Since an established COPD implies the development of systemic inflammation, a cross-sectional study design does not seem to be the best option to explore causality using conventional epidemiology. On the contrary, the prospective study offers an excellent design to explore the relationship between CRP levels and incident COPD cases and hospitalisations. Therefore, even though the results obtained from both studies are similar, the results from the prospective study are the most important in this manuscript for the association between common CRP levels and COPD; for genetically derived CRP levels, the cross-sectional study is valid indeed, because the effect of the CRP single nucleotide polymorphisms on plasma CRP levels is lifelong. The phenotype of COPD differs by the two definitions used in our study; however, the results for the two definitions were also similar. We studied only Caucasian people and our results may not necessarily apply to other ethnic groups.

In conclusion, we find that lifelong genetically elevated CRP is not associated with risk of having COPD. This suggests that the association between CRP levels and COPD is not causal. The finding is similar to those from studies in cardiovascular disease where elevated CRP is associated with cardiovascular disease, but genetic variants of CRP were not associated with disease risk. $^{20} 37$ Thus, our finding is not exclusive to COPD. It may be that for all chronic diseases associated with chronic inflammation, it is not the chronic inflammation with CRP elevation that causes disease but that the disease is driving chronic inflammation and CRP elevation.

Acknowledgements We thank laboratory technicians Hanne Damm, Dorthe Uldall Andersen and Dorthe Kjeldgaard Hansen, Department of Clinical Biochemistry, Herlev Hospital for assisting with the large-scale genotyping.

Funding Danish Heart Foundation and Danish Lung Association.

Competing interests None.

Ethics approval This study was conducted with the approval of Herlev Hospital and Danish ethics committees.

Contributors MD, JZ, AT-H and BGN did the genotyping and statistical analysis. JV and PL helped to collect the data and were involved in the statistical analysis. All investigators participated in designing the study and in the writing of the paper.

Provenance and peer review Not commissioned; externally peer reviewed. 


\section{REFERENCES}

1. Mannino DM, Buist AS. Global burden of COPD: risk factors, prevalence, and future trends. Lancet 2007;370:765-73.

2. Mathers CD, Loncar D. Projections of global mortality and burden of disease from 2002 to 2030. PLoS Med 2006;3:e442.

3. Cazzola M, MacNee W, Martinez FJ, et al. Outcomes for COPD pharmacological trials: from lung function to biomarkers. Eur Respir J 2008;31:416-69.

4. Dahl M, Nordestgaard BG. Markers of early disease and prognosis in COPD. Int J Chron Obstruct Pulmon Dis 2009;4:157-67.

5. Vestbo J, Anderson W, Coxson HO, et al. Evaluation of COPD longitudinally to identify predictive surrogate end-points (ECLIPSE). Eur Respir J 2008;31:869-73.

6. Aronson D, Roterman I, Yigla M, et al. Inverse association between pulmonary function and C-reactive protein in apparently healthy subjects. Am J Respir Crit Care Med 2006;174:626-32.

7. Dahl M, Vestbo J, Lange $\mathrm{P}$, et al. C-reactive protein as a predictor of prognosis in chronic obstructive pulmonary disease. Am J Respir Crit Care Med 2007;175:250-5.

8. de Torres JP, Cordoba-Lanus E, Lopez-Aguilar C, et al. C-reactive protein levels and clinically important predictive outcomes in stable COPD patients. Eur Respir $\mathrm{J}$ 2006;27:902-7.

9. Gan W0, Man SF, Senthilselvan A, et al. Association between chronic obstructive pulmonary disease and systemic inflammation: a systematic review and a metaanalysis. Thorax 2004;59:574-80.

10. Hancox RJ, Poulton R, Greene JM, et al. Systemic inflammation and lung function in young adults. Thorax 2007;62:1064-8.

11. Karadag $\mathbf{F}$, Kirdar $\mathrm{S}$, Karul $A B$, et al. The value of $\mathrm{C}$-reactive protein as a marker of systemic inflammation in stable chronic obstructive pulmonary disease. Eur $\mathrm{J}$ Intern Med 2008;19:104-8.

12. Man SF, Connett JE, Anthonisen NR, et al. C-reactive protein and mortality in mild to moderate chronic obstructive pulmonary disease. Thorax 2006:61:849-53.

13. Olafsdottir IS, Gislason T, Thjodleifsson B, et al. Gender differences in the association between $\mathrm{C}$-reactive protein, lung function impairment, and COPD. Int J Chron Obstruct Pulmon Dis 2007;2:635-42.

14. Pinto-Plata VM, Mullerova $\mathrm{H}$, Toso JF, et al. C-reactive protein in patients with COPD, control smokers and non-smokers. Thorax 2006:61:23-8.

15. Shaaban R, Kony S, Driss F, et al. Change in C-reactive protein levels and FEV1 decline: a longitudinal population-based study. Respir Med 2006;100:2112-20.

16. van Durme YM, Verhamme KM, Aarnoudse AJ, et al. C-reactive protein levels, haplotypes, and the risk of incident chronic obstructive pulmonary disease. Am J Respir Crit Care Med 2009;179:375-82.

17. Vestbo J. Systemic inflammation and progression of COPD. Thorax 2007;62:469-70.

18. Lowe GD, Pepys MB. C-reactive protein and cardiovascular disease: weighing the evidence. Curr Atheroscler Rep 2006:8:421-8.

19. Smith GD, Lawlor DA, Harbord R, et al. Clustered environments and randomized genes: a fundamental distinction between conventional and genetic epidemiology. PLoS Med 2007;4:e352.
20. Zacho J, Tybjaerg-Hansen A, Jensen JS, et al. Genetically elevated C-reactive protein and ischemic vascular disease. N Engl J Med 2008;359:1897-908.

21. Schnohr $\mathbf{P}$, Jensen $G$, Lange $P$, et al. The Copenhagen City Heart Study. Østerbroundersøgelsen. Tables with data from the third examination 1991-1994. Eur Heart J 2001;3(Suppl H):H1-83.

22. Dahl M, Bowler RP, Juul K, et al. Superoxide dismutase 3 polymorphism associated with reduced lung function in two large populations. Am J Respir Crit Care Med 2008;178:906-12.

23. Miller DT, Zee RY, Suk DJ, et al. Association of common CRP gene variants with CRP levels and cardiovascular events. Ann Hum Genet 2005;69:623-38.

24. Lee J, Nordestgaard BG, Dahl M. Elevated ACE activity is not associated with asthma, COPD, and COPD co-morbidity. Respir Med 2009:103:1286-92.

25. Klein J, Moeschberger ML. Refinements of the Semiparametric Proportional Hazards Model. Survival Analysis. Techniques for Censored and Truncated Data. New York USA: Springer-Verlag, Inc, 2003:295-325.

26. Clarke R, Shipley M, Lewington $S$, et al. Underestimation of risk associations due to regression dilution in long-term follow-up of prospective studies. Am J Epidemiol 1999;150:341-53.

27. MacMahon S, Peto R, Cutler J, et al. Blood pressure, stroke, and coronary heart disease. Part 1, Prolonged differences in blood pressure: prospective observational studies corrected for the regression dilution bias. Lancet 1990;335:765-74

28. Greenland S, Longnecker MP. Methods for trend estimation from summarized dose-response data, with applications to meta-analysis. Am J Epidemiol 1992; 135:1301-9.

29. Harbord RM, Lawlor DA, Smith GD. Genetically elevated C-reactive protein and vascular disease. N Engl J Med 2009;360:933-5.

30. Altman DG. Bland JM. Interaction revisited: the difference between two estimates BMJ 2003:326:219.

31. Sunyer J, Pistelli R, Plana E, et al. Systemic inflammation, genetic susceptibility and lung function. Eur Respir J 2008;32:92-7.

32. Lee TM, Lin MS, Chang NC. Usefulness of C-reactive protein and interleukin-6 as predictors of outcomes in patients with chronic obstructive pulmonary disease receiving pravastatin. Am J Cardiol 2008:101:530-5.

33. Sin DD, Lacy P, York E, et al. Effects of fluticasone on systemic markers of inflammation in chronic obstructive pulmonary disease. Am J Respir Crit Care Med 2004;170:760-5

34. Sin DD, Man SF, Marciniuk DD, et al. The effects of fluticasone with or without salmeterol on systemic biomarkers of inflammation in chronic obstructive pulmonary disease. Am J Respir Crit Care Med 2008:177:1207-14.

35. Davey SG, Ebrahim S. 'Mendelian randomization': can genetic epidemiology contribute to understanding environmental determinants of disease? Int J Epidemiol 2003;32:1-22.

36. Nitsch D, Molokhia M, Smeeth L, et al. Limits to causal inference based on Mendelian randomization: a comparison with randomized controlled trials. Am J Epidemiol 2006;163:397-403.

37. Elliott $\mathbf{P}$, Chambers JC, Zhang W, et al. Genetic Loci associated with C-reactive protein levels and risk of coronary heart disease. JAMA 2009;302:37-48.

\section{Thorax alerts}

Sign up for our electronic table of contents alerts and you will never miss new issues of Thorax when published online. Stay ahead and up to date by visiting thorax.bmj.com. 